\title{
Analytical Solution for the Sound Radiation Field of a Viscoelastically Supported Beam Traversed by a Moving Load
}

\author{
Rezgar Shakeri and Davood Younesian \\ Center of Excellence in Railway Transportation, School of Railway Engineering, Iran University of Science and Technology, \\ Tehran 16846-13114, Iran \\ Correspondence should be addressed to Davood Younesian; younesian@iust.ac.ir
}

Received 20 October 2012; Accepted 19 November 2012; Published 23 June 2014

Academic Editor: Abdolreza Ohadi

Copyright (C) 2014 R. Shakeri and D. Younesian. This is an open access article distributed under the Creative Commons Attribution License, which permits unrestricted use, distribution, and reproduction in any medium, provided the original work is properly cited.

\begin{abstract}
Sound radiation from a beam resting on a viscoelastic foundation is analytically studied when it is subjected to a moving load. The topic could cover a range of applications such as submerged floating tunnels, buried pipelines, and railway tracks. Galerkin's method is employed to obtain the transverse vibration of the beam. Based on the Rayleigh integral approach, acoustic pressure distribution around the beam is obtained in the time domain. In the second part of this paper, corresponding displacement and acoustic pressure are obtained by the use of the Rayleigh-Ritz approach in conjunction with the Laplace transform method and by the use of the Fourier transform, respectively. Durbin's numerical Laplace transform inversion scheme is eventually employed to obtain dynamic responses. A parametric study is then carried out and influences of the design parameters as well as the loading conditions on the acoustic pressure field are investigated.
\end{abstract}

\section{Introduction}

The investigation of the dynamic response of beams resting on viscoelastic foundation is a very interesting problem, with applications in various fields of engineering and technology. In particular, acoustic radiation can be found in a different array of practical applications such as submerged floating tunnels, railway tracks, and civil structures. Numerical, analytical, and experimental studies have been performed in the past decades to study the dynamic analysis of soilstructure interaction problem. Beam on elastic foundation has been studied by several authors [1-4]. Kenney [5] studied the steady-state deformation when the point load is moving with constant speed. Kargarnovin et al. [6-8] and Muscolino and Palmeri [9] investigated the response of beam on viscoelastic foundation under moving loads. Andersen et al. [10], Weitsman [11], Choros and Adams [12], and Lin and Adams [13] investigated the response of Euler-Bernoulli beams on tensionless Winkler foundations. The response of embedded railway track using the Winkler foundation model was studied by Shamalta and Metrikine [14]. Steele $[15]$ and Chen and Huang $[16,17]$ investigated the response of Timoshenko beam on Winkler foundation for a variety of beam, foundation, and loading conditions. Yang and $\mathrm{Ge}$ [18] and Senalp et al. [19] investigated the dynamic behavior of Euler-Bernoulli beam resting on viscoelastic foundation subjected to moving load by using the mode decomposition method together with the precise time integration method (MDPIM). Vlasov and Leont'ev [20] showed that the mechanical behavior of an elastic continuum can be quite accurately simulated using springs with shear interactions between them. The tensile resistance is generated in the ground due to shear strain of the soil. Basu and Kameswara Rao [21] studied the steady-state response of an infinite beam resting on a viscoelastic foundation, modeled using springs with shear interactions and subjected to a concentrated moving load with a constant velocity. In this paper, Galerkin's method is proposed to investigate the dynamic response of Euler-Bernoulli beam resting on viscoelastic foundation and shear stiffness of soil is considered. Acoustic radiation is examined by using appropriate $2 \mathrm{D}$ images of the sound field for selected foundation parameters. This paper is an invited article selected from the ISAV2012 conference proceedings. 


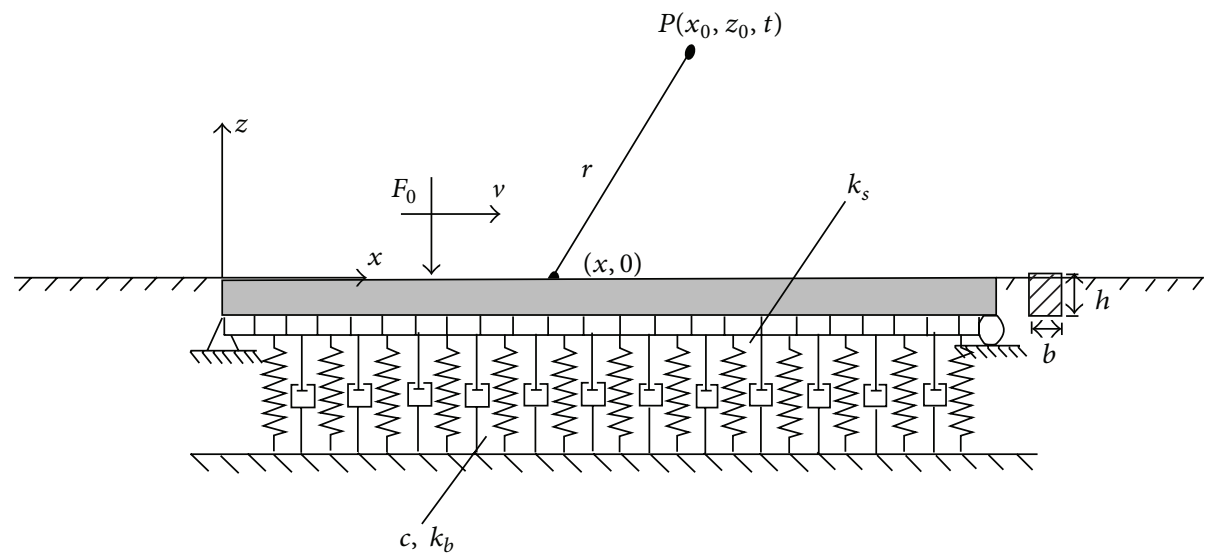

FIGURE 1: Beam on foundation under moving load.

\section{Formulation}

2.1. Analytical Method. Consider a simply supported uniform beam of length $L$, mass per unit length $\rho A$, and bending rigidity $E I$, resting on viscoelastic foundation, indicated by a bending layer coefficient $k_{b}$, a shear layer coefficient $k_{s}$, and a viscous coefficient $c$ subjected to a moving load with constant speed $v$ along the beam as shown in Figure 1. The equation of motion for the transverse vibration of the beam is given by [21]

$$
E I \frac{\partial^{4} w}{\partial x^{4}}-k_{s} \frac{\partial^{2} w}{\partial x^{2}}+k_{b} w+c \frac{\partial w}{\partial t}+\rho A \frac{\partial^{2} w}{\partial t^{2}}=-F_{0} \delta(x-v t),
$$

where $w$ is transverse deflection of the beam and $F_{0}$ is applied moving concentrated load. The displacement of the beam is expanded using the eigenfunction of the uniform beam as [22]

$$
\begin{gathered}
W_{n}(x)=\sqrt{\frac{2}{\rho A L}} \sin \frac{n \pi}{L} x, \\
w_{n}=\sqrt{\frac{E I}{\rho A}\left(\frac{n^{4} \pi^{4}}{L^{4}}+\frac{k_{s} n^{2} \pi^{2}}{E I L^{2}}+\frac{k_{b}}{E I}\right)}, \\
n=1,2, \ldots,
\end{gathered}
$$

where $\omega_{n}$ is the natural frequency of the $n$th mode and $W_{n}(x)$ is the eigenfunction. The orthogonality condition is

$$
\int_{0}^{L} \rho A W_{i}(x) W_{j}(x) d x=\delta_{i j}
$$

where $\delta_{i j}$ is Kronecker's delta. The transverse displacement can be expanded as follows:

$$
w(x, t)=\sum_{n=1}^{\infty} q_{n}(t) W_{n}(x)
$$

where $q_{n}(t)$ is the modal coordinate that must be determined by solving an ordinary differential equation. By direct substitution of expansion (4) into (1) and making use of the general orthogonality relation (3) between the distinct normal modes and the fact that the normal modes, $W_{n}(x)$, must satisfy the classical relation $E I\left(d^{4} W_{n}(x) / d x^{4}\right)-k_{s}\left(d^{2} W_{n}(x) / d x^{2}\right)+$ $k_{b} W_{n}(x)=\rho A \omega_{n}^{2} W_{n}(x)$, (1) can be reduced to

$$
\frac{d^{2} q_{n}(t)}{d t^{2}}+\frac{c}{\rho A} \frac{d q_{n}(t)}{d t}+\omega_{n}^{2} q_{n}(t)=Q_{n}(t),
$$

where $Q_{n}(t)=-\int_{0}^{L} W_{n}(x) F_{0} \delta(x-v t) d x=-F_{0} W_{n}(v t)$. The solution of (5) with the zero initial condition can be expressed as

$$
\begin{aligned}
& q_{n}(t) \\
& =\int_{0}^{t} Q_{n}(t-\tau) \\
& \quad \times \frac{2 \exp (-c \tau / 2 \rho A) \sinh \left(0.5 \tau \sqrt{(c / \rho A)^{2}-4 \omega_{n}^{2}}\right)}{\sqrt{(c / \rho A)^{2}-4 \omega_{n}^{2}}} d \tau .
\end{aligned}
$$

2.2. Rayleigh-Ritz Method. The Rayleigh-Ritz method is based on an energy or variational principle, such as those provided by the principles of virtual work or their variants, which account for the natural boundary conditions as a part of the principle. The total strain and kinetic energy and the work done by the nonconservative forces are given by [22]

$$
\begin{aligned}
U= & \frac{1}{2} \int_{0}^{L}\left(E I\left(\frac{\partial^{2} w}{\partial x^{2}}\right)^{2}+k_{b} w^{2}\right) d x \\
T= & \frac{1}{2} \int_{0}^{L} \rho A\left(\frac{\partial w}{\partial t}\right)^{2} d x \\
V= & -\int_{0}^{L} w(x, t) F_{0} \delta(x-v t) d x \\
& -\int_{0}^{L} c \frac{\partial w}{\partial t} w d x-\frac{1}{2} \int_{0}^{L} k_{s}\left(\frac{\partial w}{\partial x}\right)^{2} d x .
\end{aligned}
$$


Substituting for $U, T$, and $V$ from (7) in Hamilton's principle, we obtain

$$
\begin{aligned}
& 0=\delta \int_{0}^{t}(U-T-V) d t \\
&=\int_{0}^{t}\left\{\int _ { 0 } ^ { L } \left[E I \frac{\partial^{4} w}{\partial x^{4}}+k_{b} w+\rho A \frac{\partial^{2} w}{\partial t^{2}}+c \frac{\partial w}{\partial t}\right.\right. \\
&\left.\left.-k_{s} \frac{\partial^{2} w}{\partial x^{2}}+F_{0} \delta(x-v t)\right] \delta w d x\right\} d t .
\end{aligned}
$$

In the Ritz method, a dependent unknown (e.g., the displacement) $w(x, t)$ is approximated by a finite linear combination as the form [23]

$$
w(x, t)=\sum_{n=1}^{N} c_{n}(t) \varphi_{n}(x)+\varphi_{0}
$$

where $c_{n}(t)$ are time-dependent parameters to be determined for all times $t>0$ and $\varphi_{n}(x)$ are called the approximation functions. Since the specified geometric boundary condition is homogeneous, we have $\varphi_{0}=0$. The approximation functions $\varphi_{n}(x)$ must satisfy the simple support's condition. Direct substituting of (9) into (8) and equating coefficients of $\delta c_{m}$ to zero may advantageously be put in matrix form as

$$
\begin{gathered}
{\left[\begin{array}{ccc}
M_{11} & \cdots & M_{1 N} \\
\vdots & \ddots & \vdots \\
M_{N 1} & \cdots & M_{N N}
\end{array}\right]\left[\begin{array}{c}
c_{1}^{\prime \prime} \\
\vdots \\
c_{N}^{\prime \prime}
\end{array}\right]+\left[\begin{array}{ccc}
C_{11} & \cdots & C_{1 N} \\
\vdots & \ddots & \vdots \\
C_{N 1} & \cdots & C_{N N}
\end{array}\right]\left[\begin{array}{c}
c_{1}^{\prime} \\
\vdots \\
c_{N}^{\prime}
\end{array}\right]} \\
+\left[\begin{array}{ccc}
K_{11} & \cdots & K_{1 N} \\
\vdots & \ddots & \vdots \\
K_{N 1} & \cdots & K_{N N}
\end{array}\right]\left[\begin{array}{c}
c_{1} \\
\vdots \\
c_{N}
\end{array}\right]=\left[\begin{array}{c}
Q_{1} \\
\vdots \\
Q_{N}
\end{array}\right]
\end{gathered}
$$

where

$$
\begin{gathered}
c_{n}^{\prime \prime}=\frac{d^{2} c_{n}}{d t^{2}}, \quad c_{n}^{\prime}=\frac{d c_{n}}{d t}, \\
M_{n m}=\int_{0}^{L} \rho A \varphi_{m}(x) \varphi_{n}(x) d x, \\
C_{n m}=\int_{0}^{L} c \varphi_{m}(x) \varphi_{n}(x) d x, \\
K_{n m}=\int_{0}^{L}\left(E I \frac{d^{4} \varphi_{n}(x)}{d x^{4}}-k_{s} \frac{d^{2} \varphi_{n}(x)}{d x^{2}}+k_{b} \varphi_{n}(x)\right) \\
\times \varphi_{m}(x) d x, \\
Q_{m}(t)=-\varphi_{m}(v t) .
\end{gathered}
$$

By taking Laplace transform of (10), one obtains

$$
R(s) C(s)=Q(s),
$$

where $C(s)$ is the unknown coefficients vector and $Q(s)$ is the load vector which are given as

$$
\begin{gathered}
C(s)=\left[\bar{c}_{1}(s), \bar{c}_{2}(s), \ldots, \bar{c}_{N}(s)\right]^{T}, \\
Q(s)=\left[-\bar{\varphi}_{1}(s),-\bar{\varphi}_{2}(s), \ldots,-\bar{\varphi}_{N}(s)\right]^{T} .
\end{gathered}
$$

And the coefficient matrix $R(s)$ is rewritten as

$R(s)$

$$
=\left[\begin{array}{ccc}
s^{2} M_{11}+s C_{11}+K_{11} & \cdots & s^{2} M_{1 N}+s C_{1 N}+K_{1 N} \\
\vdots & \ddots & \vdots \\
s^{2} M_{N 1}+s C_{N 1}+K_{N 1} & \cdots & s^{2} M_{N N}+s C_{N N}+K_{N N}
\end{array}\right] .
$$

The unknown coefficient can readily be determined from

$$
C(s)=R^{-1}(s) Q(s) .
$$

In the current work, Durbin's approach [24] for numerical inversion of the Laplace transforms which involves the discretized form of the complex Laplace inversion formula $\Lambda(t)=(1 / 2 \pi j) \int_{\sigma-j \infty}^{\sigma+j \infty} \bar{\Lambda}(s) e^{s t} d s$, where $j=\sqrt{-1}$ and $\sigma$ is an arbitrary real number greater than all the real parts of the singularities of $\bar{\Lambda}(s)$ and $\bar{\Lambda}(s)$ can be $\bar{w}(x, s)$ or $\bar{p}(x, z, s)$ in the interval $\left[0,2 T_{0}\right]$, shall be adopted. Accordingly, one could readily employ expansion [25]

$$
\begin{aligned}
& \Lambda(t)= \frac{2 \exp (\sigma t)}{T_{0}} \\
& \times\left\{\frac{1}{2} \operatorname{Re}(\bar{\Lambda}(\sigma))\right. \\
&+\sum_{l=1}^{M}\left[\operatorname{Re}\left(\bar{\Lambda}\left(\sigma+j \frac{2 \pi l}{T_{0}}\right)\right) \cos \left(\frac{2 \pi l}{T_{0}} t\right)\right. \\
&\left.\left.\quad-\operatorname{Im}\left(\bar{\Lambda}\left(\sigma+j \frac{2 \pi l}{T_{0}}\right)\right) \sin \left(\frac{2 \pi l}{T_{0}} t\right)\right]\right\}
\end{aligned}
$$

where $M$ is the truncation constant and the suggested value of " $\sigma T_{0}$ " is between 5 and 10 for sufficient accuracy [24].

2.3. Acoustic Radiation. The acoustic pressure radiated from a vibration beam in an infinite baffle can be obtained by evaluating the Rayleigh surface integral where each elemental area on the beam surface is regarded as a simple point source of an outgoing wave and its contribution is added with an appropriate time delay. Referring to Figure 1, the acoustic pressure $P\left(x_{0}, z_{0}, t\right)$ at the observation point $p$ with Cartesian coordinates $\left(x_{0}, z_{0}\right)$ at time $t$ caused by the vibration of the beam is given by [26]

$$
P\left(x_{0}, z_{0}, t\right)=\frac{\rho_{0}}{2 \pi} \int_{0}^{b} \int_{0}^{L} \ddot{w}\left(x, t-\frac{r}{c_{0}}\right) \frac{1}{r} d x d y,
$$

where $\rho_{0}$ and $c_{0}$ are the mass density and wave velocity of the acoustic medium, respectively, $\ddot{w}(x, t)$ is the acceleration time history of the beam obtained previously, and $r=$ $\sqrt{\left(x-x_{0}\right)^{2}+z_{0}^{2}}$ is the distance between the observation point $p$ and the point at $(x, 0)$ on the beam surface. 
TABle 1: Properties of the UIC60 rail, foundation, load, and acoustic medium [8].

\begin{tabular}{lcc}
\hline Item & Notation & Value \\
\hline Young's modulus (steel) & $E$ & $210 \mathrm{GPa}$ \\
Mass density of beam & $\rho$ & $7850 \mathrm{~kg} / \mathrm{m}^{3}$ \\
Cross-sectional area & $A$ & $7.69 \times 10^{-3} \mathrm{~m}^{2}$ \\
Second moment of area & $I$ & $30.55 \times 10^{-6} \mathrm{~m}^{4}$ \\
Bending stiffness & $k_{b}$ & $202 \mathrm{MN} / \mathrm{m}^{2}$ \\
Viscous damping & $c$ & $141 \mathrm{kN} \mathrm{s} / \mathrm{m}^{2}$ \\
Load & $F_{0}$ & $65 \mathrm{kN}$ \\
Mass density of acoustic medium (air) & $\rho_{0}$ & $1.2 \mathrm{~kg} / \mathrm{m}^{3}$ \\
Wave velocity & $c_{0}$ & $343 \mathrm{~m} / \mathrm{s}$ \\
\hline
\end{tabular}

In addition, the pressure distribution in the acoustic fluid domain can be obtained from the solution of the wave equation as follows:

$$
\left(\frac{\partial^{2}}{\partial x^{2}}+\frac{\partial^{2}}{\partial z^{2}}\right) p(x, z, t)=\frac{1}{c_{0}^{2}} \frac{\partial^{2} p}{\partial t^{2}} .
$$

In frequency domain, (18) reduces to $\left(\left(\partial^{2} / \partial x^{2}\right)+\left(\partial^{2} / \partial z^{2}\right)+\right.$ $\left.\left(\omega^{2} / c_{0}^{2}\right)\right) p(x, z, \omega)=0$, and referring to Figure 1, the Rayleigh integral in the frequency domain [27] is $P\left(x_{0}, z_{0}, \omega\right)=$ $\left(\rho_{0} / 2 \pi\right) \int_{0}^{b} \int_{0}^{L} \ddot{w}(x, \omega)(\exp (i k r) / r) d x d y$, where $k=\omega / c_{0}$ and $\ddot{w}(x, \omega)$ is the acceleration of the beam in the frequency domain. Taking Laplace transform of (18) leads to $\left(\left(\partial^{2} / \partial x^{2}\right)+\right.$ $\left.\left(\partial^{2} / \partial z^{2}\right)+\left(-s^{2} / c_{0}^{2}\right)\right) \bar{p}(x, z, s)=0$, and then, the Rayleigh integral in the Laplace domain is

$$
\bar{P}\left(x_{0}, z_{0}, s\right)=\frac{s^{2} \rho_{0}}{2 \pi} \int_{0}^{b} \int_{0}^{L} \bar{w}(x, s) \frac{\exp (i \bar{k} r)}{r} d x d y,
$$

where $\bar{k}=j s / c_{0}$ and $\bar{w}(x, s)=\sum_{n=0}^{\infty} q_{n}(s) W_{n}(x)$ is the displacement of the beam in the Laplace domain where $W_{n}(x)$ is given by (2) and $q_{n}(s)$ is obtained from (5). Finally, the acoustic pressure can be calculated by (16). In the following section, the comparison between these two methods is considered.

\section{Numerical Results}

A parametric study is directed in this section to investigate the effects of different parameters on the acoustic pressure field radiated from the vibrating beam series of numerical simulations that have been carried out and some of the numerical results are presented as a few samples in this section. The physical and geometrical properties of the beam and acoustic medium are listed in Table 1 [8].

Figure 2 exhibits the midspan displacement of the beam with respect to the time. Because of the foundation under the beam, the vibration around the maximum deflection is zero. The maximum deflection will occur at the location of the load. Furthermore, excellent agreement of the present result and [19] for load velocity $v=50 \mathrm{~m} / \mathrm{s}$ is obtained. Figure 3 displays the comparison between the results of

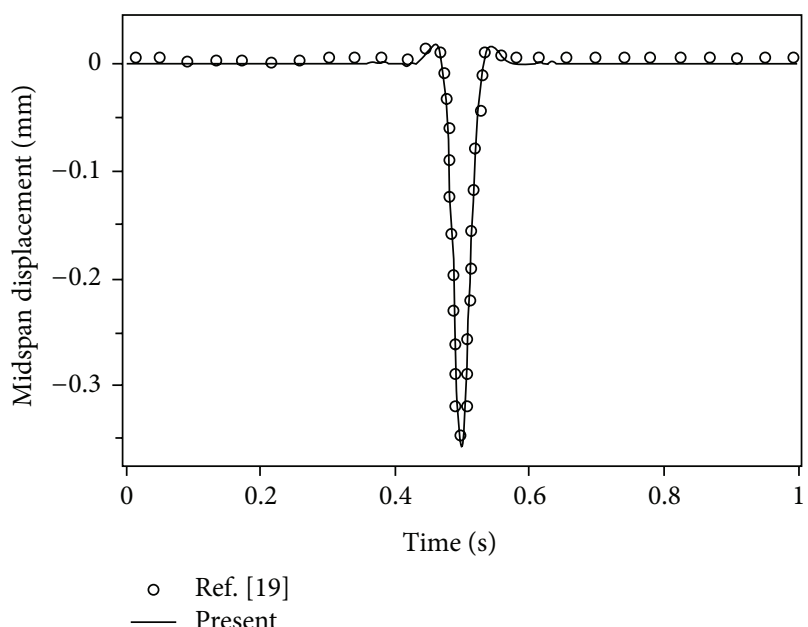

Figure 2: Comparison of the displacement response with [19].

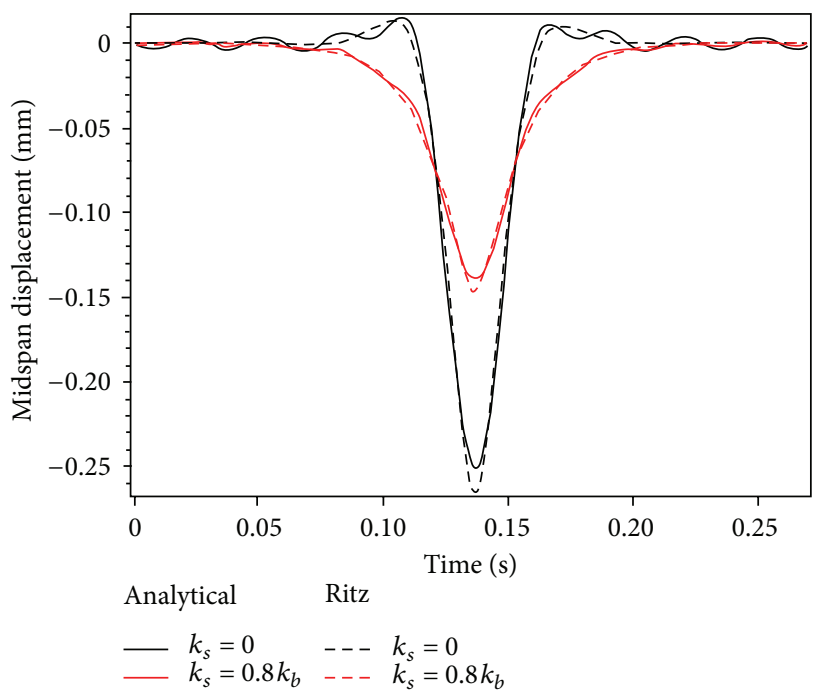

FIgURE 3: Comparison of analytical and Rayleigh-Ritz methods.

the analytical and Rayleigh-Ritz methods for selected load velocity $v=55 \mathrm{~m} / \mathrm{s}$ and for a beam extended $15 \mathrm{~m}$ in longitudinal direction. The results show a good agreement between these two methods. Taking shear layer coefficient into consideration, the maximum deflection of the beam will decrease when the shear layer coefficient is increased due to increment of natural frequencies of the beam.

Figure 4 shows the acoustic pressure response at two selected points and for $k_{s}=0, k_{s}=0.8 k_{b}$ when the load passes through the beam. The acoustic pressure decreases as the viscoelastic and bending stiffness and height of selected points increase. Also for $k_{s}=0.8 k_{b}$, an interesting reduction of the first radiated pressure wave from the vibrating beam is seen at $t_{0}=z_{0} / c_{0}=(0.014 \& 0.058)$ sec.

Figure 5 displays the acoustic pressure response and comparison between two methods for $t \geq r / c_{0}$. The results show good agreement between two equations. Equation (17) exhibits instabilities for time interval of $0 \leq t<r / c_{0}$; 

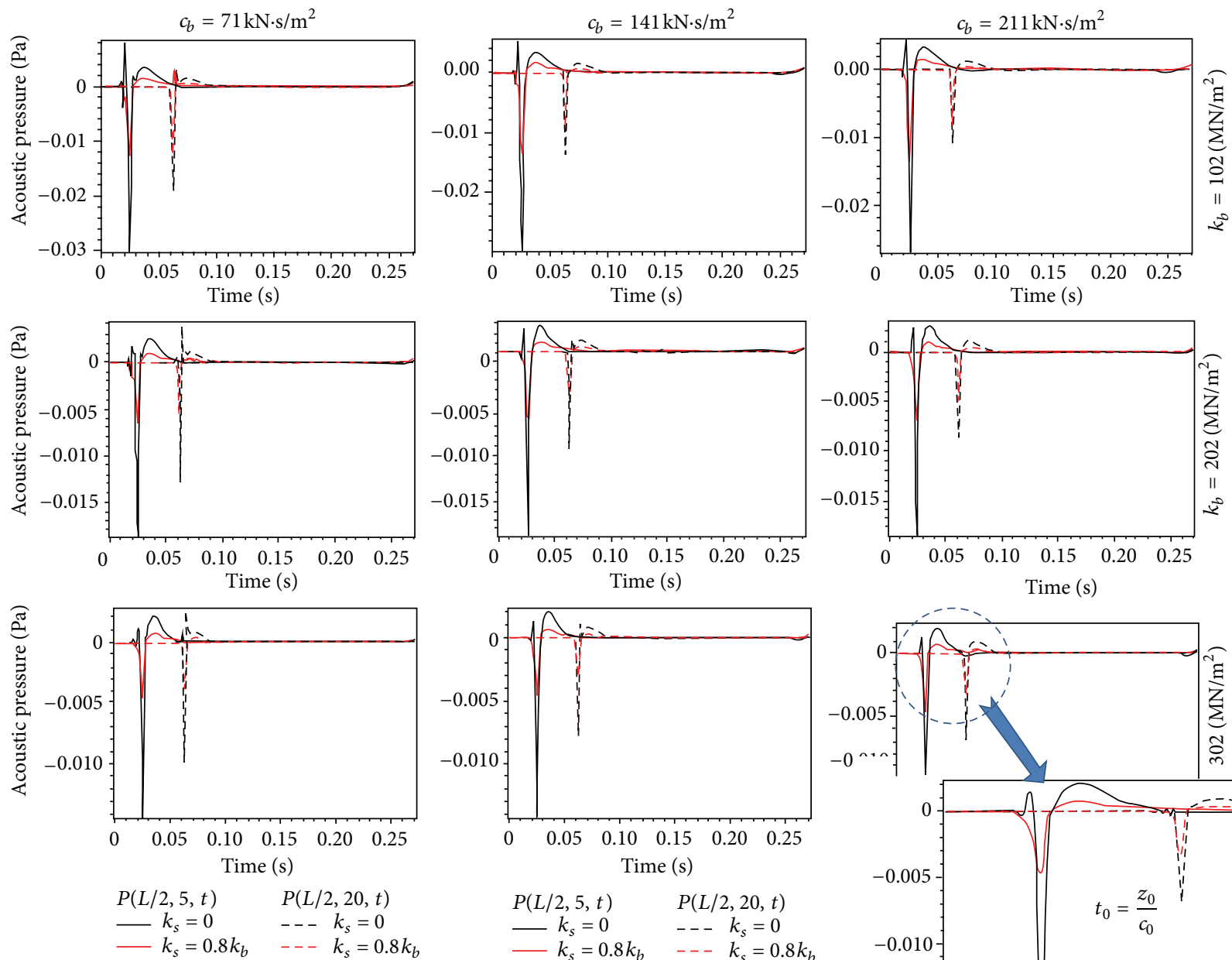

$$
\begin{array}{ll}
P(L / 2,5, t) & P(L / 2,20, t) \\
-k_{s}=0 & ---k_{s}=0 \\
-k_{s}=0.8 k_{b} & ---k_{s}=0.8 k_{b}
\end{array}
$$

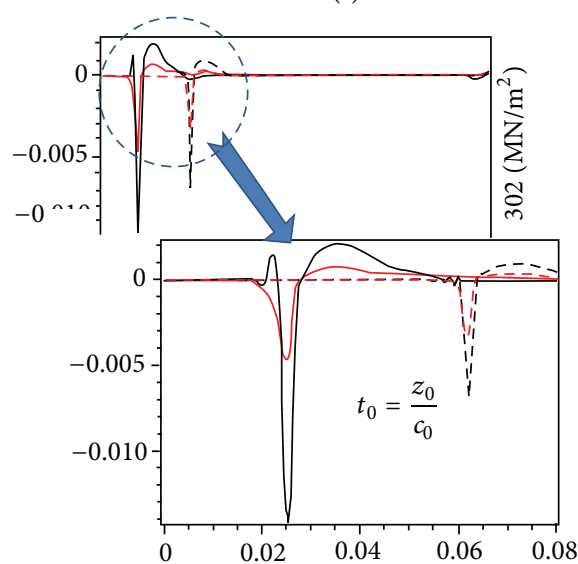

Figure 4: Acoustic pressure at different heights (midspan).

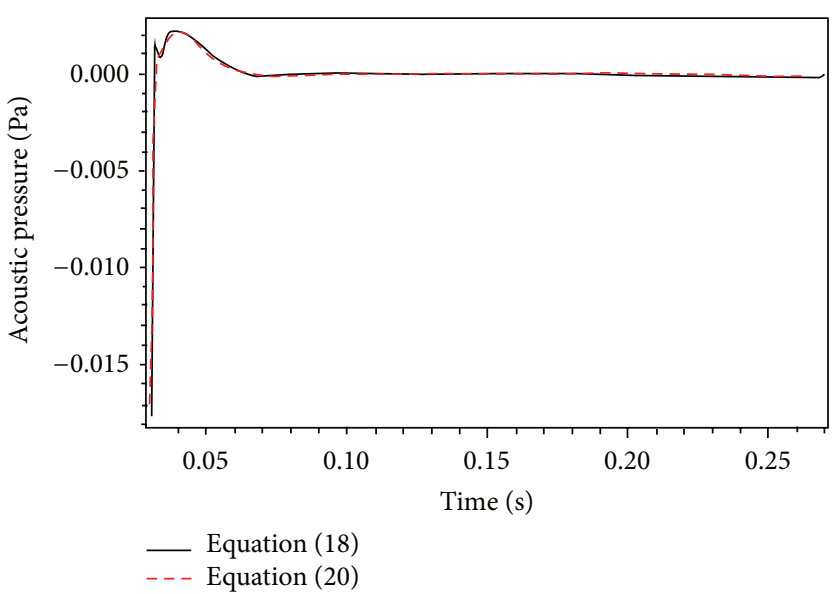

FIGURE 5: Comparison between two methods for calculating acoustic radiation. 

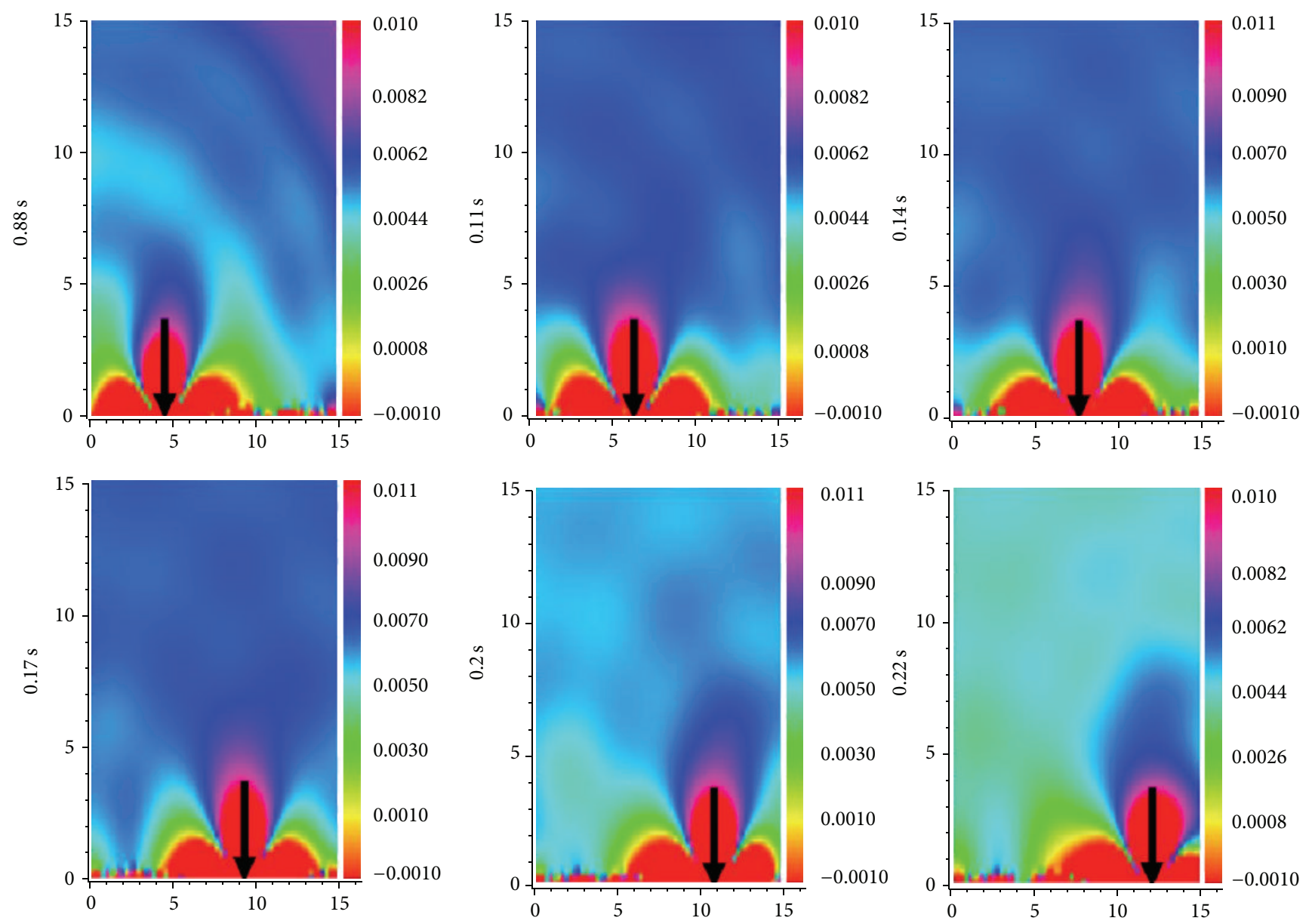

Figure 6: Acoustic pressure field ( $\mathrm{Pa})$ along the beam $(15 \mathrm{~m})$ at different positions of moving load.

therefore, it must be calculated by numerical techniques such as standard trapezoidal rule [26]. On the other hand, robustness of (19) was seen in both of the mentioned time spans.

Figure 6 shows the series of time snapshots of acoustic pressure distribution while the moving load is passing on the beam for shear layer coefficient $k_{s}=0.8 k_{b}$, length of the beam $L=15$, bending layer coefficient $k_{b}=202 \mathrm{MN} / \mathrm{m}^{2}$, viscous coefficient $c=141 \mathrm{kN} \cdot \mathrm{sec} / \mathrm{m}^{2}$, and typical vehicle load $(65 \mathrm{kN})$. The acoustic pressure fields around the beam show that maximum distribution pressure appears around the location of the moving load which is a result of maximum deflection of the beam.

\section{Conclusion}

In this paper, transient response of simply supported, EulerBernoulli beam resting on viscoelastic foundation subjected to a moving load is studied. Based on the Rayleigh integral and dynamic response of the beam, the acoustic pressure distribution around the beam was obtained for the first time. The time responses of the beam with and without shear stiffness effects of the soil are presented for various foundations of moving load. The effects of $k_{s}=0$ and $k_{s}=0.8 k_{b}$ can be easily observed from Figure 4 . The most important observations are summarized as follows:

(i) the beam structural vibration is observed to radiate sound into acoustic medium. In particular, increasing the magnitude of foundation is seen to decrease the acoustic pressure;

(ii) increasing the height of selected points decreases the acoustic pressure radiation, while the time response keeps the same trend;

(iii) the delay of the first acoustic pressure wave radiated from the beam is easily seen;

(iv) the contours of acoustic pressure fields around the beam show that maximum distribution pressure appears around the location of the moving load which is a result of maximum deflection of the beam.

\section{Conflict of Interests}

The authors declare that there is no conflict of interests regarding the publication of this paper. 


\section{References}

[1] M. Hetenyi, in Beams on Elastic Foundations, vol. 16 of Scientific Series, The University of Michigan Press, University of Michigan Studies, Ann Arbor, Mich, USA, 1946.

[2] L. Andersen, S. R. K. Nielsen, and P. H. Kirkegaard, "Finite element modelling of infinite Euler beams on Kelvin foundations exposed to moving loads in convected co-ordinates," Journal of Sound and Vibration, vol. 241, no. 4, pp. 587-604, 2001.

[3] D. Thambiratnam and Y. Zhuge, "Dynamic analysis of beams on an elastic foundation subjected to moving loads," Journal of Sound and Vibration, vol. 198, no. 2, pp. 149-169, 1996.

[4] L. Fryba, Vibration of Solids and Structures Under Moving Loads, Thomas Telford, London, UK, 1999.

[5] J. T. Kenney, "Steady-state vibrations of beam on elastic foundation for moving load," Journal of Applied Mechanics, vol. 21, pp. 359-364, 1954.

[6] M. H. Kargarnovin, D. Younesian, D. J. Thompson, and C. J. C. Jones, "Response of beams on nonlinear viscoelastic foundations to harmonic moving loads," Computers and Structures, vol. 83, no. 23-24, pp. 1865-1877, 2005.

[7] M. H. Kargarnovin and D. Younesian, "Dynamics of Timoshenko beams on Pasternak foundation under moving load," Mechanics Research Communications, vol. 31, no. 6, pp. 713-723, 2004.

[8] D. Younesian and M. H. Kargarnovin, "Response of the beams on random Pasternak foundations subjected to harmonic moving loads," Journal of Mechanical Science and Technology, vol. 23, no. 11, pp. 3013-3023, 2010.

[9] G. Muscolino and A. Palmeri, "Response of beams resting on viscoelastically damped foundation to moving oscillators," International Journal of Solids and Structures, vol. 44, no. 5, pp. 1317-1336, 2007.

[10] L. Andersen, S. R. K. Nielsen, and R. Iwankiewicz, "Vehicle moving along an infinite beam with random surface irregularities on a Kelvin foundation," Journal of Applied Mechanics, Transactions ASME, vol. 69, no. 1, pp. 69-75, 2002.

[11] Y. Weitsman, "Onset of separation between a beam and a tensionless elastic foundation under a moving load," International Journal of Mechanical Sciences, vol. 13, no. 8, pp. 707-711, 1971.

[12] J. Choros and G. G. Adams, "Steadily moving load on an elastic beam resting on a tensionless winkler foundation," Journal of Applied Mechanics, Transactions ASME, vol. 46, no. 1, pp. 175180, 1979.

[13] L. Lin and G. G. Adams, "Beam on tensionless elastic foundation," Journal of Engineering Mechanics, vol. 113, no. 4, pp. 542553, 1987.

[14] M. Shamalta and A. V. Metrikine, "Analytical study of the dynamic response of an embedded railway track to a moving load," Archive of Applied Mechanics, vol. 73, no. 1-2, pp. 131-146, 2003.

[15] C. R. Steele, "The Timoshenko beam with a moving Load," Journal of Applied Mechanics, vol. 35, no. 3, pp. 481-488, 1968.

[16] Y.-H. Chen and Y.-H. Huang, "Dynamic stiffness of infinite Timoshenko beam on viscoelastic foundation in moving coordinate," International Journal for Numerical Methods in Engineering, vol. 48, no. 1, pp. 1-18, 2000.

[17] Y.-H. Chen and Y.-H. Huang, "Dynamic characteristics of infinite and finite railways to moving loads," Journal of Engineering Mechanics, vol. 129, no. 9, pp. 987-995, 2003.
[18] Y. Yang and X. Ge, "A new method for vibration response of beam on foundation under moving load," International Journal of Modern Physics B, vol. 22, no. 31-32, pp. 5615-5620, 2008.

[19] A. D. Senalp, A. Arikoglu, I. Ozkol, and V. Z. Dogan, "Dynamic response of a finite length euler-bernoulli beam on linear and nonlinear viscoelastic foundations to a concentrated moving force," Journal of Mechanical Science and Technology, vol. 24, no. 10, pp. 1957-1961, 2010.

[20] V. Z. Vlasov and U. N. Leont'ev, Beams, Plates and Shells on Elastic Foundations, Israel Program for Scientific Translations, Jerusalem, Israel, 1966.

[21] D. Basu and N. S. V. Kameswara Rao, "Analytical solutions for Euler-Bernoulli beam on visco-elastic foundation subjected to moving load," International Journal for Numerical and Analytical Methods in Geomechanics, vol. 37, no. 8, pp. 945-960, 2013.

[22] S. S. Rao, Vibration of Continuous Systems, Wiley, New York, NY, USA, 2007

[23] J. N. Reddy, Theory and Analysis of Elatic Plates and Shells, CRC Press, New York, NY, USA, 2007.

[24] F. Durbin, "Numerical inversion of laplace transforms: an efficient improvement to dubner and abate's method," Computer Journal, vol. 17, no. 4, pp. 371-376, 1974.

[25] S. M. Hasheminejad, R. Shakeri, and S. Rezaei, "Vibro-acoustic response of an elliptical plate-cavity coupled system to external shock loads," Applied Acoustics, vol. 73, no. 8, pp. 757-769, 2012.

[26] F. T. K. Au and M. F. Wang, "Sound radiation from forced vibration of rectangular orthotropic plates under moving loads," Journal of Sound and Vibration, vol. 281, no. 3-5, pp. 1057-1075, 2005.

[27] M. C. Junger and D. Feit, Sound, Structures and Interaction, MIT Press, Cambridge, Mass, USA, 1986. 

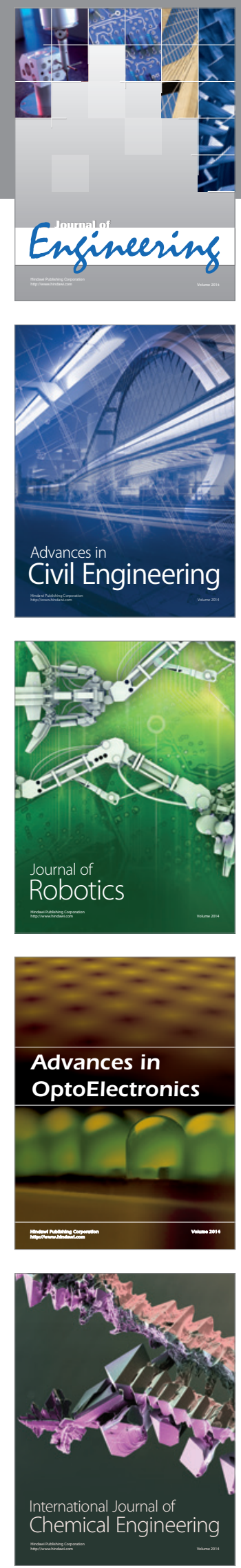

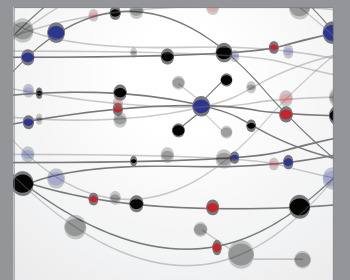

The Scientific World Journal
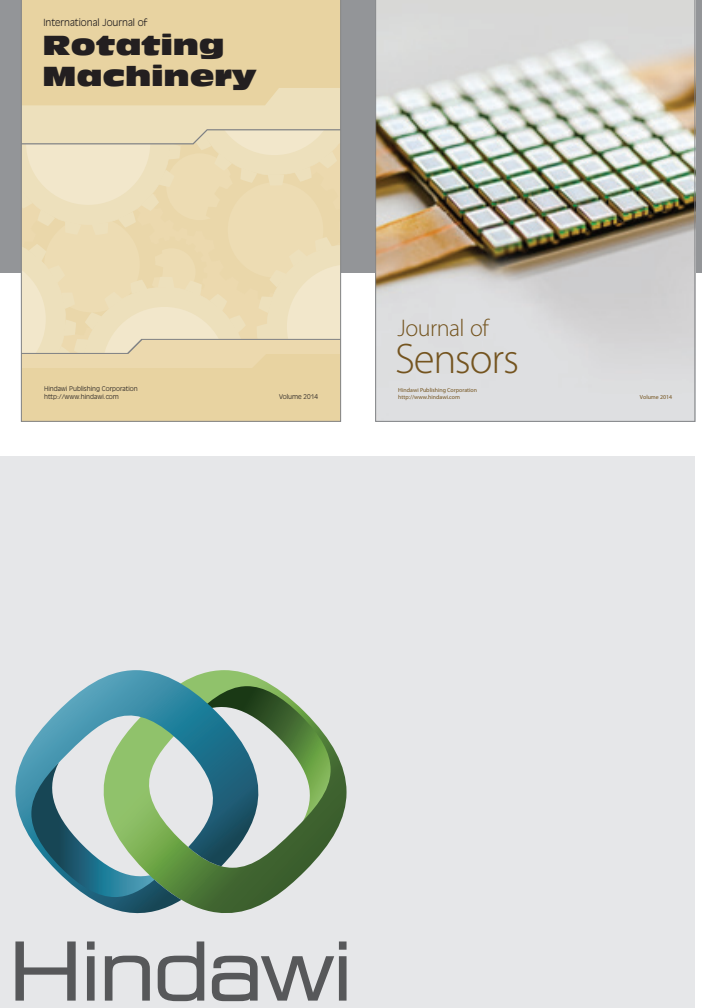

Submit your manuscripts at http://www.hindawi.com
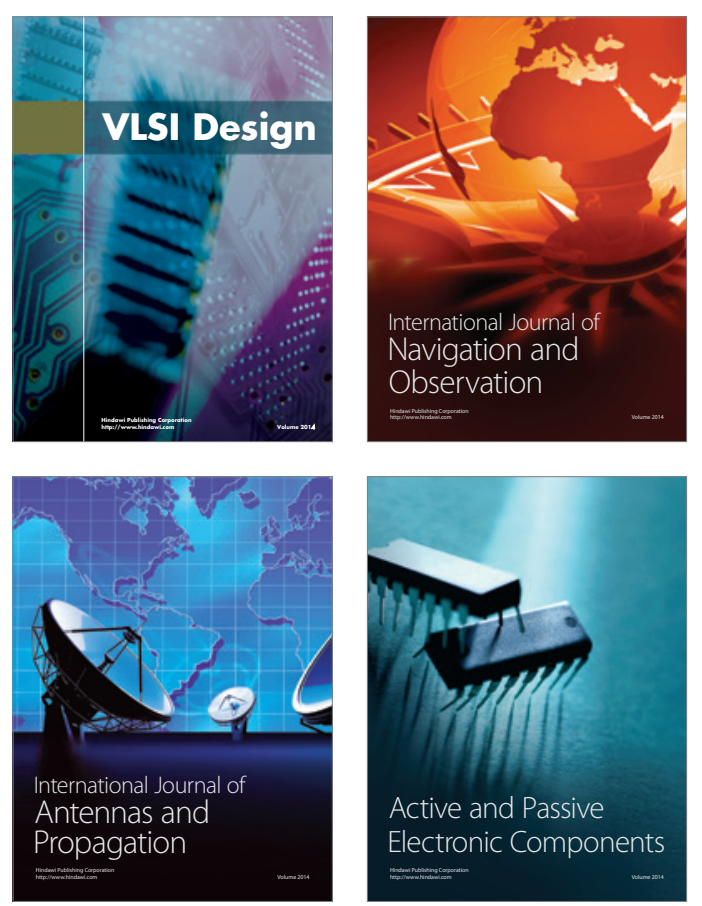
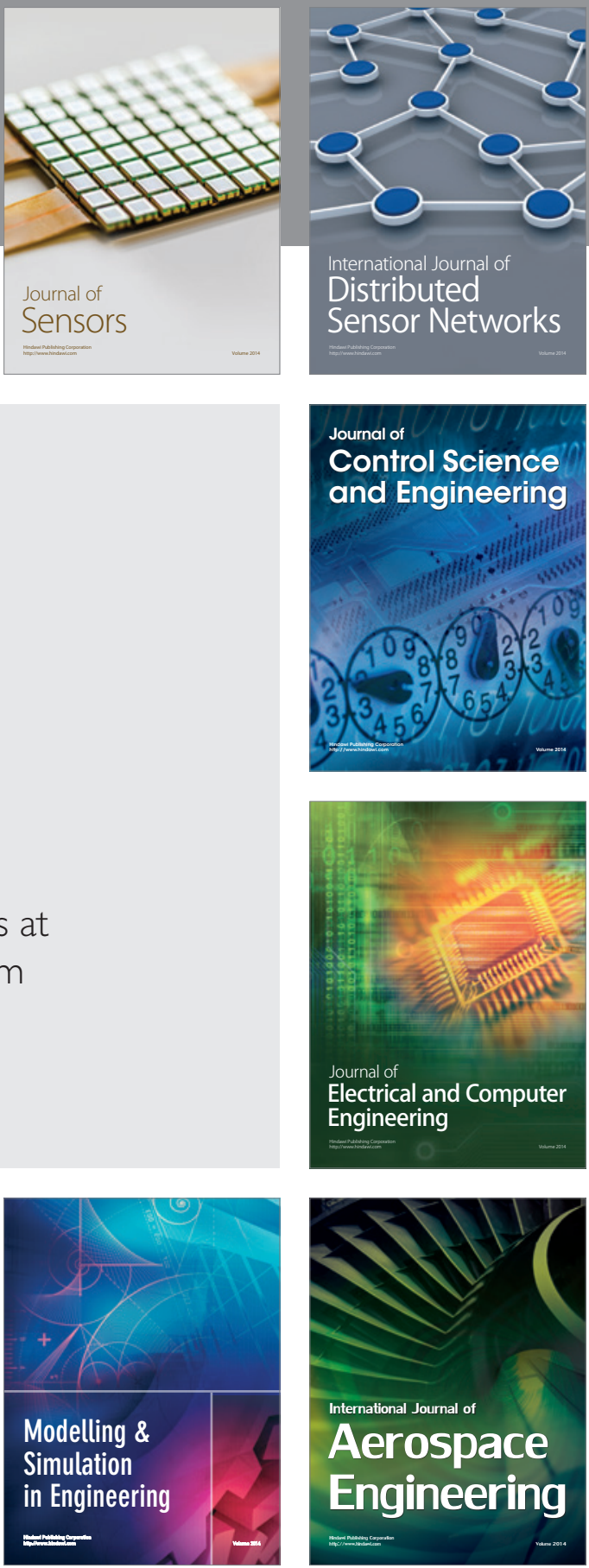

Journal of

Control Science

and Engineering
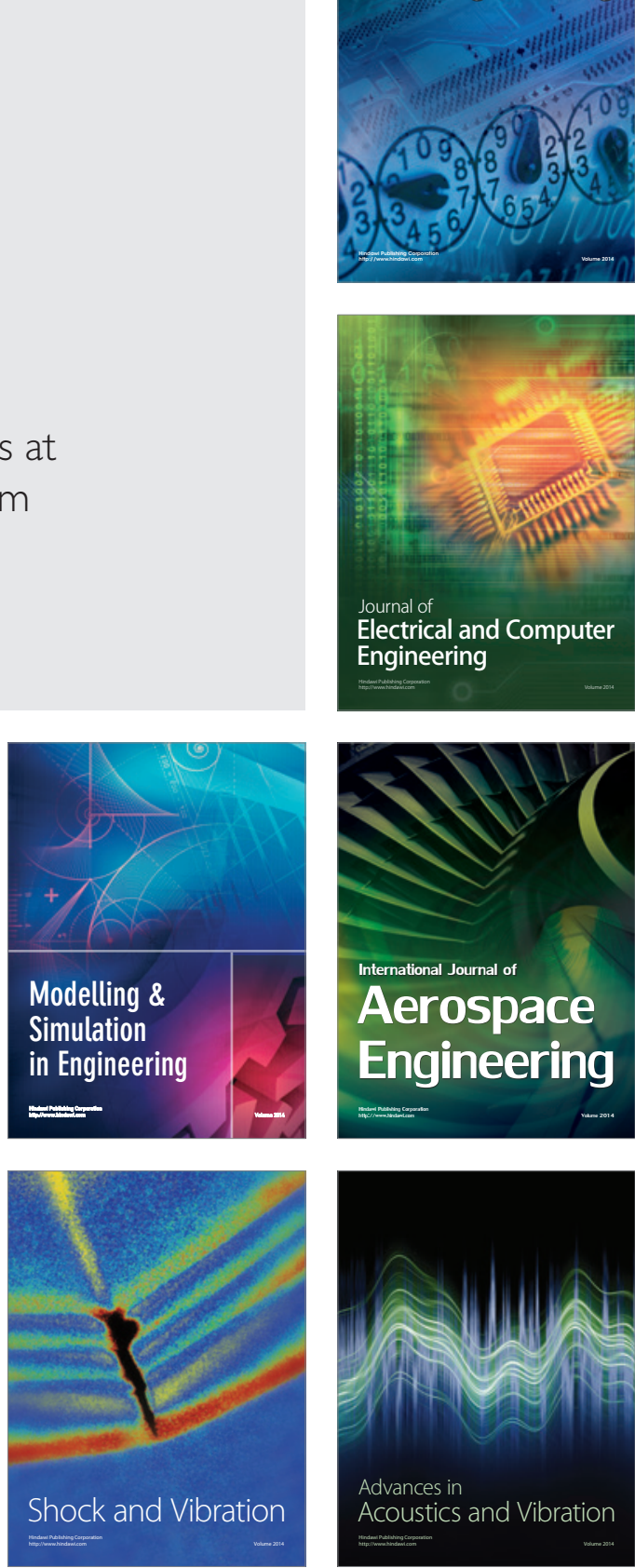